
\title{
25 Research Soure \\ Clinical Characteristics and Outcomes of Idiopathic Membranous Nephropathy with Glomerular Igm Deposits
}

\section{Yu-Zhu Xu}

Sun Yat-sen University First Affiliated Hospital https://orcid.org/0000-0001-6017-1571

Na-Ya Huang

Sun Yat-sen University First Affiliated Hospital

Ya-Gui Qiu

Sun Yat-sen University First Affiliated Hospital

\section{Xiong-Hui Chen}

Sun Yat-sen University First Affiliated Hospital

\section{Zhi-Jian Li}

Sun Yat-sen University First Affiliated Hospital

\section{Wei Chen}

Sun Yat-sen University First Affiliated Hospital

Xin Wang ( $\square$ wangxin8@mail.sysu.edu.cn )

Sun Yat-sen University First Affiliated Hospital

\section{Research Article}

Keywords: IgM deposition, Idiopathic membranous nephropathy, Clinicopathological features, Renal outcomes.

Posted Date: August 5th, 2021

DOl: https://doi.org/10.21203/rs.3.rs-732093/v1

License: (a) (1) This work is licensed under a Creative Commons Attribution 4.0 International License. Read Full License 


\section{Abstract}

\section{Background}

Glomerular IgM deposition is commonly shown in idiopathic membranous nephropathy (IMN), but the clinicopathological features and outcomes of IMN with IgM deposition are unclear.

\section{Methods}

This single-center prospective cohort study enrolled 210 patients with biopsy-proven IMN from January 2016 to December 2018. Clinicopathological features, treatment responses, and kidney outcomes were compared between patients with and without IgM deposition.

\section{Results}

76 (36.2\%) patients shown glomerular IgM deposition. Patients with IgM deposition were younger ( $45 \pm 13.296$ vs. $50.59 \pm 13.65$ years, $P=0.006$ ), had a higher estimated glomerular filtration rate (eGFR) (100.03 [81.31-111.37] vs. 92.67 [74.71-106.63] $\left.\mathrm{mL} / \mathrm{min} / 1.73 \mathrm{~m}^{2}, \mathrm{P}=0.041\right)$, and had a lower proportion of nephrotic syndrome $(60.5 \%$ vs. $75.4 \%, P=0.024)$ at the time of kidney biopsy. Patients with IgM deposition had a significantly higher proportion of focal segmental glomerular sclerosis (FSGS) lesions ( $27.6 \%$ vs. $13.4 \%, P=0.011)$ and $\mathrm{C} 1 \mathrm{q}$ deposition $(72.4 \%$ vs. $57.5 \%, \mathrm{P}=0.032)$. Although the treatments and initial treatment responses were comparable, patients with glomerular IgM deposition had a significantly greater proportion of eGFR decline of $\geq 5 \mathrm{~mL} / \mathrm{min} / 1.73 \mathrm{~m}^{2}$ per year (log-rank test, $P<0.001$ ) and eGFR decrease of $\geq 10 \%$ from baseline (log-rank test, $P=0.003$ ). Cox regression analysis showed that $\lg M$ deposition was an independent risk factor of eGFR decline of $\geq 5 \mathrm{~mL} / \mathrm{min} / 1.73 \mathrm{~m}^{2}$ per year (HR, 2.442; $95 \% \mathrm{Cl}, 1.550-3.848, \mathrm{P}<0.001)$ and eGFR decline by $\geq 10 \%$ from baseline (HR, $2.629 ; 95 \% \mathrm{Cl}, 1.578-$ $4.385, \mathrm{P}<0.001)$ during follow-up.

\section{Conclusions}

Glomerular IgM deposition is associated with younger age, more severe FSGS lesions and C1q deposition, and worse renal outcomes in IMN.

\section{Background}

Idiopathic membranous nephropathy (IMN) is the most common cause of nephrotic syndrome in adults [1, 2], and has become the most common primary glomerular disease following IgA nephropathy (IgAN) in China [3]. IMN is mediated by a variety of autoantibodies against podocytes and is clinically characterized by proteinuria or nephrotic syndrome and pathologically characterized by granular deposits of IgG and C3 subepithelial deposits, diffuse thickening of the basement membrane, and extensive fusion of podocyte foot processes $[1,4,5]$. 
Complement activation is confirmed to be an indispensable link in the pathogenesis of IMN [1, 6-8]. However, the main pathogenic antibodies of IMN are the anti-M-type phospholipase A2 receptor (PLA2R) antibody [9] and the thrombospondin type-1 domain-containing 7A (THSD7A) antibody [10], which are dominated by the IgG4 subtype. Although some studies have suggested that IgG4 may mediate podocyte injury by activating complement through the lectin pathway $[11,12]$, most studies indicate that IgG4 has a very limited capability to activate the complement system, and it is believed that IgG4 cannot activate complement through the classical and alternative pathways $[13,14]$.

Traditionally, deposition of other types of immune complexes (IgM, IgA, C1q) in the glomeruli is usually considered to be a feature of secondary membranous nephropathy [15]. In clinical practice, however, we have found that many patients who have IMN with positive anti-PLA2R antibodies also have IgM deposition in the glomeruli. Whether glomerular IgM deposition has an impact on the clinicopathological features and outcomes of IMN is currently unknown. In this study, we investigated the clinicopathological characteristics and clinical outcomes of IMN with glomerular IgM deposition.

\section{Methods}

\section{Study population}

This study involved consecutively enrolled patients with biopsy-proven IMN from 1 January 2016 to 31 December 2018 in the First Affiliated Hospital of Sun Yat-sen University. Patients with known secondary MN such as that caused by a tumor, rheumatic immune disease (e.g., systemic lupus erythematosus, Sjogren's syndrome, rheumatoid arthritis, antineutrophil cytoplasmic antibody-associated vasculitis), infectious-related nephritis (e.g., hepatitis B-related nephritis, hepatitis C, syphilis), a clear history of nephrotoxic drug use or history of heavy metal exposure, and diabetic nephropathy were excluded. The pathological data and clinical data of 210 patients with IMN who met the inclusion criteria were collected and followed up until 31 December 2019 for at least 6 months or until they developed end-stage renal disease or died. This research complied with the Declaration of Helsinki and was approved by the ethics committee of Sun Yat-sen University (EC No. 2019482).

\section{Clinical and pathological data}

Nephrotic syndrome is defined as a urinary protein concentration of $>3.5 \mathrm{~g}$ per 24 hours and a serum albumin concentration of $<30 \mathrm{~g} / \mathrm{L}$. The estimated glomerular filtration rate (eGFR) was calculated using the formula established by the Chronic Kidney Disease Epidemic Collaboration [16]:

eGFR $=144 \times \times($ if female: serum creatinine $\leq 62(0.7))$

eGFR $=144 \times \times($ if female: serum creatinine $>62(0.7))$

eGFR $=141 \times \times($ if male: serum creatinine $\leq 80(0.9))$

eGFR $=141 \times(\times($ if male: serum creatinine $>80(0.9))$ 
Glomerular membranous nephropathy lesions were classified into four stages according to the Ehrenreich-Churg staging system. Chronic tubulointerstitial injury lesions were graded semiquantitatively from 0 to 2 according to the percentage of tubular atrophy injury: $0, \leq 5 \% ; 1,6 \%-25 \%$; and $2,>25 \%$. The degree of interstitial fibrosis was defined as absent, mild, moderate, or severe. The fluorescence intensity of the IgG, IgM, IgA, C3, C1q, and IgG subclass was determined as negative or positive. Non-physiological glomerulosclerosis was defined as a proportion of glomerulosclerosis exceeding $(($ age / 2$)-10) \times 100 \%[17,18]$.

\section{Treatment and follow-up}

The use of corticosteroids and immunosuppressive agents was in compliance with the 2012 KDIGO guidelines [19]. Complete remission was defined as proteinuria of $\leq 0.3 \mathrm{~g}$ per 24 hours and a serum albumin concentration of $\geq 35 \mathrm{~g} / \mathrm{L}$ regardless of creatinine clearance [20]. Partial remission was defined as a reduction in proteinuria of $\geq 50 \%$ from baseline plus final proteinuria of 0.3 to $3.5 \mathrm{~g}$ per 24 hours and a serum albumin level of $\geq 30 \mathrm{~g} / \mathrm{L}$ regardless of creatinine clearance [20]. Clinical remission included complete remission and partial remission. Non-response, deterioration, and remission did not reach the

level of partial remission; all were defined as no response. The primary endpoint event was renal function decline of $\geq 5 \mathrm{~mL} / \mathrm{min} / 1.73 \mathrm{~m}^{2}$ per year and a $\geq 10 \%$ decline of eGFR from baseline. The secondary endpoint event was clinical remission.

\section{Statistical analysis}

Statistical analysis was performed using SPSS version 25 software (IBM Corp., Armonk, NY, USA), and P $<0.05$ was considered statistically significant. Normally distributed variables are expressed as mean \pm standard deviation, and non-normally distributed continuous variables are expressed as median with interquartile range. Categorical variables are expressed as absolute value and percentage. For continuous variables, comparisons between two groups were performed using Student's $t$ test for normally distributed data and the Wilcoxon rank-sum test for nonparametric data. For categorical variables, the chi-square test was used (Fisher's exact test was used when the theoretical frequency was $<5$ ). The correlation of IgM deposition with focal segmental glomerular sclerosis (FSGS) lesions and C1q deposition was tested using Pearson's chi-square analysis. Kaplan-Meier survival analysis was used to compare survival between the IgM-positive and -negative groups, and a Cox proportional hazards model was used to analyze the correlation between IgM deposition and renal outcomes.

\section{Results}

\section{Patients' baseline clinical and pathological characteristics}

Among the 210 patients with biopsy-proven IMN, 126 (60.0\%) were male and their median age was 50.00 (40.00-59.25) years. The urinary protein excretion rate was 6.25 (3.09-9.16) g per 24 hours, the median serum albumin concentration was $23.85(19.80-28.18) \mathrm{g} / \mathrm{L}$, the median serum creatinine concentration 
was $76.00(62.00-95.00) \mu \mathrm{mol} / \mathrm{L}$, and the median eGFR was $94.57(76.55-108.50) \mathrm{mL} / \mathrm{min} / 1.73 \mathrm{~m}^{2}$ (Table 1).

In total, 39 (18.6\%) patients presented without glomerulosclerosis, 39 (18.6\%) patients showed FSGS lesions, and 15 (7.1\%) patients exhibited crescent formation. Additionally, 209 (99.5\%) patients presented with granular staining of $\operatorname{lgG}$, and IgG subclass staining was available in $139(66.2 \%)$ patients. Of these 139 patients, 60 (43.2\%) had IgG2 deposition, 135 (97.1\%) had IgG4 deposition, and 124 (89.2\%) had predominant IgG4 deposition. Moreover, 76 (36.2\%) patients presented with IgM deposition and 181 (86.2\%) patients showed C3 staining along the glomerular capillary walls. A total of 139 patients had available glomerular PLA2R staining; of them, 131 (94.2\%) showed positive PLA2R staining in the glomerular basement membrane (Table 2).

For the initial treatment, $128(46.2 \%)$ patients were treated conservatively and $149(53.8 \%)$ were treated with immunosuppressants. Of these 149 patients, 69 (46.3\%) were treated with corticosteroids only, 71 $(47.7 \%)$ were treated with corticosteroids plus calcineurin inhibitors, and $9(6.0 \%)$ were treated with corticosteroids plus cyclophosphamide (Table 1).

\section{Clinicopathological characteristics of patients with IMN with IgM deposition}

Among the 210 patients with IMN, 76 had IgM deposition in the glomeruli. Of these 76 patients, 54 had glomerular PLA2R staining, and 52 (96.3\%) were positive. Compared with patients without glomerular IgM deposition, patients with IgM deposition were younger ( $45 \pm 13.296$ vs. $50.59 \pm 13.65, P=0.006$ ), had a higher eGFR (100.03 [81.31-111.37] vs. 92.67 [74.71-106.63], $P=0.041)$, and showed a lower proportion of nephrotic syndrome $(60.5 \%$ vs. $75.4 \%, \mathrm{P}=0.024)$ at the time of kidney biopsy (Table 3$)$.

Pathologically, patients who had IMN with IgM deposition had a higher proportion of concurrent FSGS lesions $(27.6 \%$ vs. $13.4 \%, P=0.011)$ and $\mathrm{C} 1 \mathrm{q}$ deposition $(72.4 \%$ vs. $57.5 \%, \mathrm{P}=0.032)$ (Table 4$)$, and there was no statistically significant difference in treatments or treatment responses between the two groups (Table 3).

\section{Correlation of IgM deposition with FSGS lesions and C1q and C3 deposition}

Of the 39 patients with FSGS lesions, 23 (59.0\%) presented with C1q deposition and 35 (89.7\%) had C3 deposition. All patients with FSGS + C1q deposition had C3 deposition. Moreover, correlation analysis showed a significant correlation between IgM deposition and FSGS lesions $(r=0.175, P=0.011), C 1 q$ deposition $(r=0.148, P=0.032)$, and FSGS + C1q deposition $(r=0.244, P<0.001)$ (Table 5).

\section{IgM deposition and renal outcomes}

Kaplan-Meier survival curves preliminarily showed that compared with patients without glomerular IgM deposition, more patients with glomerular IgM deposition had an eGFR decline of $\geq 5 \mathrm{~mL} / \mathrm{min} / 1.73 \mathrm{~m}^{2}$ per year (log-rank test, $P<0.001$ ) and an eGFR decrease of $\geq 10 \%$ from baseline (log-rank test, $P=0.003$ ) (Figure 2). 
After adjusting for age, sex, urinary protein level, eGFR, glomerulosclerosis, FSGS lesions, crescents, chronic interstitial tubular injury, hyalinization, stage of membranous nephropathy, C1q deposition, treatment regimen, and treatment response factors, multiple Cox regression analysis showed that the risk of an eGFR decline of $\geq 5 \mathrm{~mL} / \mathrm{min} / 1.73 \mathrm{~m}^{2}$ per year and an eGFR decline of $\geq 10 \%$ from baseline was 2.442 times higher ( $95 \%$ confidence interval, $1.550-3.848, \mathrm{P}<0.001)$ and 1.853 times higher $(95 \%$ confidence interval, $1.059-3.240, \mathrm{P}=0.031$ ) in patients with than without IgM deposition during the follow-up period (Table 6).

\section{Discussion}

This study involved 210 adult patients with biopsy-proven IMN from 2016 to 2018, of whom 76 (36.2\%) had IgM deposition in the glomeruli. We found that patients who had IMN with glomerular IgM deposition were younger, had better renal function, and had a lower proportion of nephrotic syndrome than those without IgM deposition at the time of kidney biopsy. Pathologically, patients who had IMN with glomerular IgM deposition had more C1q deposition and more FSGS lesions. Although there was no significant difference in treatment regimens and clinical remission between the two groups, the risk of renal function deterioration was higher in the IgM deposition group, and Cox multivariate regression analysis showed that IgM deposition in the glomeruli was an independent risk factor for renal function deterioration in patients with IMN.

Similar to our study, previous studies have shown that among patients with FSGS [21] and IgAN [22], those with IgM deposition were younger (FSGS positive vs. negative, 24.5 [18.8-39.0] vs. 46.5 [26.064.0] years; IgAN moderate vs. no or trace, $31.71 \pm 10.21$ vs. $34.82 \pm 11.03$ years), but patients with IgM deposition showed heavier proteinuria. The reason for these differences may be that IMN is a podocyte lesion disease, which itself is characterized by massive proteinuria, and the podocyte induced massive proteinuria covered up the enhanced proteinuria caused by IgM deposition damage.

Our study also showed that patients with IgM deposition presented with more glomerular C1q deposition and FSGS lesions. Meanwhile, 59.0\% (23/39) of patients with FSGS lesions had C1q deposition. Moreover, FSGS lesions and C1q deposition were significantly correlated with IgM deposition. Consistent with our study, it has been shown that patients who have IgAN with IgM deposition have a higher C1q deposition rate and more severe Oxford classification (statistically significant differences in mesangial proliferation, endocapillary proliferation, segmental sclerosis, and crescents) [22]. There should be no significant difference in immune complex deposition in the glomeruli in patients with typical FSGS lesions, thin basement membrane nephropathy, and mild glomerular lesions, but one study showed that patients with FSGS lesions had a significantly higher IgM deposition rate than patients with thin basement membrane nephropathy and mild glomerular lesions ( $55.4 \%$ vs. $30.0 \%$ ) [23]. In addition, other studies have shown that patients with IgM nephropathy who present with only IgM deposits in the mesangial area are more likely to progress to FSGS lesions as confirmed by repeat renal biopsy [24-26]. In patients with primary FSGS lesions, the presence of concurrent IgM and C3 deposition has been shown to be an independent risk factor for renal insufficiency (hazard ratio, 5.67) [21]. Some studies have 
suggested that IgM deposition in the mesangial region is a transitional state between minimal change disease and FSGS $[25,27]$. All of the above studies suggested that glomerular IgM deposition may be associated with FSGS lesions.

IgM is mainly secreted and synthesized by plasma cells in the spleen and lymph nodes, accounting for $5 \%$ to $10 \%$ of the total serum immunoglobulins. IgM exists as a pentamer and is the largest immunoglobulin in the human circulatory system. A commonly accepted view is glomerular lgM deposition represents passive trapping of the large IgM macromolecule within areas of sclerosis. It was recently proposed that in kidney disease, antibodies mainly exert their pathogenic effects by activating complement. Activation of C1q is the first step in activation of the classical complement pathway. C1q has six sites that can bind to immunoglobulin molecules, and it is activated only when more than two sites are bound. As a pentamer, IgM has a higher ability to activate C1q than monomer-form IgG [2830]. Numerous studies have confirmed that IgM deposition in the glomeruli is often accompanied by $\mathrm{C}$, C4, and C1q deposition [22, 31-33]. Animal models have demonstrated that IgM deposited within the glomerulus mediates glomerulosclerosis by activating the classical and alternative complement pathways [29,34]. Panzer et al. [35] established a mouse model of non-sclerotic glomerulonephritis and found that special IgM that recognizes phospholipids became bound to glomerular epitopes and contributed to the progression of glomerular damage. Strassheim et al. [29] found that depletion of peritoneal B lymphocytes by hypotonic shock had no effect on plasma IgM levels in a mouse model of adriamycin-induced FSGS but could prevent both glomerular IgM and C3 deposition; additionally, they found that $\mathrm{C} 3$ and IgM co-localized within the glomeruli. These results suggest that the IgM deposited in the glomeruli may be secreted by innate immune B-1 a cells that recognize non-protein antigens $[29,36$, 37].

Our study also showed no difference in short-term treatment responses between patients with and without IgM deposition under similar treatment ( $40.8 \%$ vs. $45.5 \%, 26.3 \%$ vs. $18.7 \%$, and $32.9 \%$ vs. $35.8 \%$ for complete remission, partial remission, and non-response, respectively; $P=0.852)$. However, renal outcomes were worse in the IgM deposition group, and IgM deposition was an independent risk factor for poor renal function outcomes in patients with IMN. Similarly, a study of 106 patients with primary FSGS showed no statistically significant difference in the initial treatment response or relapse rate between patients with and without IgM deposition when the initial treatment was comparable, but the proportion of refractory nephrotic syndrome (defined as no response to treatment or steroid-dependency) was higher in patients with IgM deposition ( $34.0 \%$ vs. $15.6 \%, P=0.04$; the proportion of non-response to treatment was $18.9 \%$ vs. $11.1 \%, P=0.43$ ), and IgM deposition was an independent risk factor for refractory disease (odds ratio, 3.97) and renal progression (hazard ratio, 4.75) [21]. Similarly, patients who had minimal change disease with IgM deposition showed a higher proportion of steroid-resistant disease $(45.5 \% \mathrm{vs}$. $41.0 \%, P=0.05)$, and more patients progressed to renal failure $(10.4 \%$ vs. $1.7 \%, P=0.05)$ [38]. In patients with IgAN, more severe IgM deposition is associated with heavier proteinuria (3.38 \pm 3.14 vs. $2.52 \pm 3.01$ g per 24 hours), more frequent use of immunosuppressive therapy (35.71\% vs. $20.48 \%)$, a lower complete remission rate ( $45.54 \%$ vs. $63.39 \%$ ), and worse renal outcomes (hazard ratio, 1.69) [22]. The presence of FSGS lesions is used as an Oxford classification indicator to predict renal outcomes in patients with 
IgAN [39]. Therefore, we hypothesize that glomerular IgM deposition in patients with IMN may mediate FSGS lesions by activating complement and accelerating renal function deterioration.

This study had several limitations. First, this was a single-center observational study, and the samples might be biased. Second, this was a cohort study and lacked direct evidence to establish a causal relationship between IgM deposition and the pathological changes and prognosis of IMN. Third, we did not have complete information on anti-PLA2R and THSD7A antibodies in the study patients, and no further analysis of the effects of serum anti-PLA2R antibodies on the treatment response and renal function prognosis could be performed. Finally, this study had a relatively short follow-up period; longterm follow-up is needed in further studies.

\section{Conclusion}

IgM deposition in the glomeruli is an independent risk factor for decreased renal function in patients with IMN.

\section{Declarations}

\section{Acknowledgment}

Sincere thanks should be given to all of our colleagues at The First Affiliated Hospital of Sun Yat-sen University, especially the Department of Nephrology. We thank Angela Morben, DVM, ELS, from Liwen Bianji (Edanz) (www.liwenbianji.cn/), for editing the English text of a draft of this manuscript.

Funding: This study was partly supported by Operational Grant of Guangdong Provincial Key Laboratory [No. 2017B030314019]; Key Laboratory of National Health Commission; Key Laboratory of Nephrology, Guangdong Province, Guangzhou, China [No. 2002B60118]; Guangdong Natural Science Foundation [grant No.2020A1515010241]; the Natural Science Foundation of Guangdong Province, China [Grant No.2017A030310199]; and 5010 Clinical Program of Sun Yat-sen University [No.2017007].

Conflicts of interest: The authors declare that there is no conflict of interest.

Availability of data and material: No applicable.

Code availability: No applicable.

Authors' contributions: Yu-Zhu Xu: Writing-Original draft preparation, Investigation. Na-Ya Huang: Original draft preparation, Investigation. Ya-Gui Qiu: Visualization, Investigation. Xiong-Hui Chen \& Zhi-Jian Li: Resources, Conceptualization. Wei Chen \& Xin Wang: Writing- Reviewing and Editing, Project administration.

Ethics approval: This research complied with the Declaration of Helsinki and was approved by the ethics committee of Sun Yat-sen University [EC No. 2019482]. 
Consent to participate: This study used the data obtained in the previous clinical practice, which has little risk to the subjects, so informed consent was waived, which was approved by the ethics committee of Sun Yat-sen University.

Consent for publication: The data are anonymous, and informed consent was therefore waived.

\section{References}

1. Ronco P, Debiec H: Pathophysiological advances in membranous nephropathy: time for a shift in patient's care. The Lancet 2015, 385(9981):1983-92.https://dx.doi.org/10.1016/s01406736(15)60731-0.

2. Zhu P, Zhou F D, Wang S X, Zhao M H, Wang H Y: Increasing frequency of idiopathic membranous nephropathy in primary glomerular disease: a 10-year renal biopsy study from a single Chinese nephrology centre. Nephrology (Carlton) 2015, 20(8):560-6.https://dx.doi.org/10.1111/nep.12542.

3. Xu X, Wang G, Chen N et al: Long-Term Exposure to Air Pollution and Increased Risk of Membranous Nephropathy in China. J Am Soc Nephrol 2016, 27(12):3739-

46.https://dx.doi.org/10.1681/ASN.2016010093.

4. Zeng $\mathrm{CH}$, Chen $\mathrm{H}$ M, Wang R S et al: Etiology and clinical characteristics of membranous nephropathy in Chinese patients. Am J Kidney Dis 2008, 52(4):691-

8.https://dx.doi.org/10.1053/j.ajkd.2008.06.006.

5. Debiec H, Ronco P: Immunopathogenesis of membranous nephropathy: an update. Semin Immunopatho/ 2014, 36(4):381-97.https://dx.doi.org/10.1007/s00281-014-0423-y.

6. Cunningham $P \mathrm{~N}$, Quigg $\mathrm{R} \mathrm{J}$ : Contrasting roles of complement activation and its regulation in membranous nephropathy. J Am Soc Nephrol 2005, 16(5):1214-

22.https://dx.doi.org/10.1681/ASN.2005010096.

7. $\mathrm{WH}, \mathrm{DB} \mathrm{H}, \mathrm{S} \mathrm{H}$ et al: Production of nephrotic syndrome in rats by Freund's adjuvants and rat kidney suspensions. 1959, 100(4):660-4.https://dx.doi.org/10.3181/00379727-100-24736.

8. DJ S, S B, MP M, investigation C W J T J o c: A new role for complement in experimental membranous nephropathy in rats. 1980, 66(6):1339-50.https://dx.doi.org/10.1172/jci109987.

9. LH B, RG B, G L et al: M-type phospholipase A2 receptor as target antigen in idiopathic membranous nephropathy. N Engl J Med 2009, 361(1):11-21.https://dx.doi.org/10.1056/NEJMoa0810457.

10. Tomas N M, Beck L H, Jr., Meyer-Schwesinger $C$ et al: Thrombospondin type-1 domain-containing 7A in idiopathic membranous nephropathy. N Engl J Med 2014, 371(24):2277-

87.https://dx.doi.org/10.1056/NEJMoa1409354.

11. Lhotta K, Wurzner R, Konig P: Glomerular deposition of mannose-binding lectin in human glomerulonephritis. Nephrol Dial Transplant 1999, 14(4):881-

6.https://dx.doi.org/10.1093/ndt/14.4.881.

12. Hayashi N, Okada K, Matsui $Y$ et al: Glomerular mannose-binding lectin deposition in intrinsic antigen-related membranous nephropathy. Nephrol Dial Transplant 2018, 33(5):832- 
40.https://dx.doi.org/10.1093/ndt/gfx235.

13. JS v d Z, P v S, Clinical A R J, immunology e: Inhibition of complement activation by IgG4 antibodies. 1986, 64(2):415-22.https://dx.doi.org/undefined.

14. RC A, Immunology S J J: IgG4 breaking the rules. 2002, 105(1):9-

19.https://dx.doi.org/10.1046/j.0019-2805.2001.01341.x.

15. Huang C C, Lehman A, Albawardi A et al: IgG subclass staining in renal biopsies with membranous glomerulonephritis indicates subclass switch during disease progression. Mod Pathol 2013, 26(6):799-805.https://dx.doi.org/10.1038/modpathol.2012.237.

16. Kong X, Ma Y, Chen J et al: Evaluation of the Chronic Kidney Disease Epidemiology Collaboration equation for estimating glomerular filtration rate in the Chinese population. Nephrol Dial Transplant 2013, 28(3):641-51.https://dx.doi.org/10.1093/ndt/gfs491.

17. Kaplan C, Pasternack B, Shah H, Gallo G J T A j o p: Age-related incidence of sclerotic glomeruli in human kidneys. 1975, 80(2):227-34.

18. Kasiske B J K i: Relationship between vascular disease and age-associated changes in the human kidney. 1987, 31(5):1153-9.https://dx.doi.org/10.1038/ki.1987.122.

19. Group K D I G O K G W: Improving Global Outcomes (KDIGO) Glomerulonephritis Work Group. KDIGO Clinical Practice Guideline for Glomerulonephritis Kidney Inter 2012(Suppl 2):139 『274.

20. Fervenza F C, Appel G B, Barbour S J et al: Rituximab or Cyclosporine in the Treatment of Membranous Nephropathy. N Engl J Med 2019, 381(1):3646.https://dx.doi.org/10.1056/NEJMoa1814427.

21. Zhang Y M, Gu Q H, Huang J et al: Clinical Significance of IgM and C3 Glomerular Deposition in Primary Focal Segmental Glomerulosclerosis. Clin J Am Soc Nephrol 2016, 11(9):15829.https://dx.doi.org/10.2215/CJN.01190216.

22. Tan L, Tang Y, Pei G Q et al: Mesangial IgM deposition predicts renal outcome in patients with IgA nephropathy: a multicenter, observational study. Clin Exp Med 2021.https://dx.doi.org/10.1007/s10238-021-00703-1.

23. Pacic A, Senjug $P$, Bacalja J et al: IgM as a novel predictor of disease progression in secondary focal segmental glomerulosclerosis. Croat Med J 2017, 58(4):281-

91.https://dx.doi.org/10.3325/cmj.2017.58.281.

24. Myllymäki J, Saha H, Mustonen J, Helin H, Pasternack A: clinical picture and long-term prognosis. 2003, 41(2):343-50.https://dx.doi.org/10.1053/ajkd.2003.50042.

25. Zeis P, Kavazarakis E, Nakopoulou L et al: Glomerulopathy with mesangial IgM deposits: long-term follow up of 64 children. 2001, 43(3):287-92.https://dx.doi.org/10.1046/j.1442-200x.2001.01396.x.

26. Saha H, Mustonen J, Pasternack A, Helin H J A j o n: Clinical follow-up of 54 patients with IgMnephropathy. 1989, 9(2):124-8.https://dx.doi.org/10.1159/000167950.

27. Ji-Yun $Y$, Melvin T, Sibley R, Michael A J K i: No evidence for a specific role of IgM in mesangial proliferation of idiopathic nephrotic syndrome. 1984, 25(1):100- 
6.https://dx.doi.org/10.1038/ki.1984.14.

28. Czajkowsky D, Shao Z J P o t N A o S o t U S o A: The human IgM pentamer is a mushroom-shaped molecule with a flexural bias. 2009, 106(35):14960-5.https://dx.doi.org/10.1073/pnas.0903805106.

29. Strassheim D, Renner B, Panzer $S$ et al: IgM contributes to glomerular injury in FSGS. J Am SoC Nephrol 2013, 24(3):393-406.https://dx.doi.org/10.1681/ASN.2012020187.

30. McMullen M E, Hart M L, Walsh M C et al: Mannose-binding lectin binds IgM to activate the lectin complement pathway in vitro and in vivo. Immunobiology 2006, 211(10):75966.https://dx.doi.org/10.1016/j.imbio.2006.06.011.

31. Lawler W, Williams G, Tarpey P, Mallick N J J o c p: IgM associated primary diffuse mesangial proliferative glomerulonephritis. 1980, 33(11):1029-38.https://dx.doi.org/10.1136/jcp.33.11.1029.

32. Hsu $\mathrm{H}$, Chen $\mathrm{W}$, Lin $\mathrm{G}$ et al: Clinical and immunopathologic study of mesangial IgM nephropathy: report of 41 cases. 1984, 8(3):435-46.https://dx.doi.org/10.1111/j.1365-2559.1984.tb02355.x.

33. Cavallo T, Johnson M J N: Immunopathologic study of minimal change glomerular disease with mesangial IgM deposits. 1981, 27(6):281-4.https://dx.doi.org/10.1159/000182071.

34. Thurman J M, Wong M, Renner B et al: Complement Activation in Patients with Focal Segmental Glomerulosclerosis. PLoS One 2015, 10(9):e0136558.https://dx.doi.org/10.1371/journal.pone.0136558.

35. Panzer S E, Laskowski J, Renner B et al: IgM exacerbates glomerular disease progression in complement-induced glomerulopathy. Kidney Int 2015, 88(3):528-

37.https://dx.doi.org/10.1038/ki.2015.120.

36. Baumgarth N: The double life of a B-1 cell: self-reactivity selects for protective effector functions. Nat Rev Immunol 2011, 11(1):34-46.https://dx.doi.org/10.1038/nri2901.

37. Hamaguchi Y, Uchida J, Cain D W et al: The peritoneal cavity provides a protective niche for B1 and conventional B lymphocytes during anti-CD20 immunotherapy in mice. $J$ Immuno/ 2005, 174(7):4389-99.https://dx.doi.org/10.4049/jimmunol.174.7.4389.

38. Al Romaili D M, Al-Hussain T O, Awad H S et al: Clinical significance of IgM deposition in pediatric minimal change disease. Int J Pediatr Adolesc Med 2019, 6(4):14650.https://dx.doi.org/10.1016/j.ijpam.2019.09.001.

39. El Karoui K, Hill G S, Karras A et al: Focal segmental glomerulosclerosis plays a major role in the progression of IgA nephropathy. II. Light microscopic and clinical studies. Kidney Int 2011, 79(6):64354.https://dx.doi.org/10.1038/ki.2010.460.

\section{Tables}

Table 1. Clinical characteristics of patients with IMN 


\begin{tabular}{|c|c|}
\hline Parameters & $n=210$ \\
\hline Age (years) & $50.00(40.00-59.25)$ \\
\hline Gender (M), n (\%) & $126(60.0)$ \\
\hline hypertension, n (\%) & $75(35.7)$ \\
\hline diabetes, n (\%区 & $23(11.0)$ \\
\hline Nephritic syndrome, n (\%) & $147(70.0)$ \\
\hline Proteinuria (g/24h) & $6.25(3.09-9.16)$ \\
\hline Serum albumin $(\mathrm{g} / \mathrm{L})$ & $23.85(19.80-28.18)$ \\
\hline Serum creatinine $(\mu \mathrm{mol} / \mathrm{L})$ & $76.00(62.00-95.00)$ \\
\hline eGFR (ml/min /1.73) & $94.57(76.55-108.50)$ \\
\hline eGFR $₫ 60 \mathrm{ml} / \mathrm{min} / 1.73$ & $23(11.0)$ \\
\hline eGFR $₫ 30 \mathrm{ml} / \mathrm{min} / 1.73$ & $8(3.8)$ \\
\hline \multicolumn{2}{|l|}{ Treatments } \\
\hline ACEI/ARBs only, n (\%) & $83(39.5)$ \\
\hline Immunosuppressive therapies, $\mathrm{n}(\%)$ & $127(60.5)$ \\
\hline Corticosteroids only, n (\%) & $57 / 127(44.9)$ \\
\hline corticosteroids + CNIs, n (\%) & $61 / 127(48.0)$ \\
\hline corticosteroids + CTX, n (\%) & $9 / 127(7.1)$ \\
\hline Follow-up duration (months) & $25.00(15.75-37.00)$ \\
\hline \multicolumn{2}{|l|}{ Treatment responses } \\
\hline Complete remission, n (\%) & $92 / 210(43.8)$ \\
\hline Partial remission, n (\%) & $45 / 210(21.4)$ \\
\hline No response, n (\%) & $73 / 210(34.8)$ \\
\hline death, $\mathrm{n}(\%)$ & $5 / 210(2.4)$ \\
\hline ESRD(eGFR $15 \mathrm{ml} / \mathrm{min} / 1.73, \mathrm{n}(\%)$ & $1 / 210(0.5)$ \\
\hline eGFR decline $\geq 50 \%$, n (\%) & $9 / 210(4.3)$ \\
\hline eGFR decline $\geq 30 \%$, n (\%) & $26 / 210(12.4)$ \\
\hline Creatinine double, $n(\%)$ & $3 / 210(1.4)$ \\
\hline
\end{tabular}


IMN, idiopathic membranous nephropathy; $M$, male; eGFR, estimated glomerular filtration rate; ACEI/ARB, angiotensin-converting enzyme inhibitors/angiotensin receptor blockers; CNIs, calcineurin inhibitors (including cyclophosphamide A and tacrolimus); CTX, cyclophosphamide; ESRD, end-stage renal disease

Table 2. Pathological characteristics of patients with IMN 


\begin{tabular}{|c|c|}
\hline Parameters & $\mathrm{N}=210$ \\
\hline Global sclerosis, n (\%) & $147(70.0)$ \\
\hline Non-physiological global sclerosisÊ, n (\%) & $41(19.5)$ \\
\hline FSGS, n (\%) & $39(18.6)$ \\
\hline Crescent, n (\%) & $15(7.1)$ \\
\hline \multicolumn{2}{|l|}{ Chronic interstitial fibrosis } \\
\hline absent, n (\%) & $62(29.5)$ \\
\hline mild, n (\%) & $129(61.4)$ \\
\hline moderate +severe, $\mathrm{n}(\%)$ & $19(9.0)$ \\
\hline \multicolumn{2}{|l|}{ Tubular atrophy } \\
\hline $0-5 \%, n(\%)$ & $149(71.0)$ \\
\hline $6 \%-25 \%, n(\%)$ & $50(23.8)$ \\
\hline$凶 25 \%, \mathrm{n}(\%)$ & $11(5.2)$ \\
\hline Vascular hyalinosis, n (\%) & $91(43.3)$ \\
\hline \multicolumn{2}{|l|}{ Membranous Churg's stagesÖ } \\
\hline 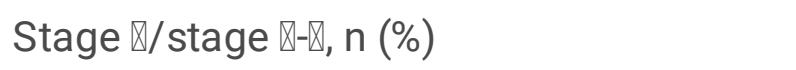 & $98(46.7)$ \\
\hline Stage $\rrbracket, n(\%)$ & $103(49.0)$ \\
\hline 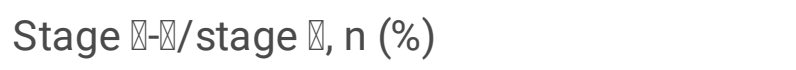 & $9(4.3)$ \\
\hline \multicolumn{2}{|l|}{ Immunofluorescence } \\
\hline IgG deposit, n (\%) & $209(99.5)$ \\
\hline IgG subclassification, n (\%) & $139(66.2)$ \\
\hline $\lg \mathrm{g} 1, \mathrm{n}(\%)$ & $130 / 139(95.3)$ \\
\hline $\lg \mathrm{g} 2, \mathrm{n}(\%)$ & $60 / 139(43.2)$ \\
\hline $\lg G 3, \mathrm{n}(\%)$ & 75/139 (54.0) \\
\hline $\operatorname{lgG} 4, \mathrm{n}(\%)$ & $135 / 139(97.1)$ \\
\hline IgG4 dominant, n (\%) & $124 / 139(89.2)$ \\
\hline $\lg A, n(\%)$ & $62(29.5)$ \\
\hline $\lg M, n(\%)$ & $76(36.2)$ \\
\hline C3, n (\%) & $181(86.2)$ \\
\hline
\end{tabular}

Page 14/21 


\begin{tabular}{|ll|}
\hline $\mathrm{C} 1 \mathrm{q}, \mathrm{n}(\%)$ & $132(66.2)$ \\
\hline Glomerular PLA2R fluorescence, $\mathrm{n}(\%)$ & $139(62.4)$ \\
\hline PLA2R positive, $\mathrm{n}(\%)$ & $131 / 139(94.2)$ \\
\hline
\end{tabular}

IMN, idiopathic membranous nephropathy; FSGS, focal segmental glomerular sclerosis; PLA2R, M-type phospholipase $\mathrm{A} 2$ receptor

ÊDefined as proportion of glomerulosclerosis exceeding ((age / 2) - 10) $\times 100 \%$

ÖGlomerular IMN lesions were classified according to the Ehrenreich-Churg classification criteria

Table 3. Clinical characteristics of patients with IMN with and without IgM deposition 


\begin{tabular}{|c|c|c|c|}
\hline Parameters & $\begin{array}{l}\text { With IgM deposition } \\
n=76\end{array}$ & $\begin{array}{l}\text { Without IgM deposition } \\
\mathrm{n}=134\end{array}$ & $P$ value \\
\hline Gender (male/female) & $42 / 34$ & $84 / 50$ & 0.291 \\
\hline Age (years $\rrbracket$ & $45 \pm 13.296$ & $50.59 \pm 13.65$ & 0.006 \\
\hline Proteinuria (g/24h) & $4.50(2.26-9.04)$ & $6.58(3.51-9.5)$ & 0.149 \\
\hline Serum albumin(g/L) & $24.55 \pm 6.802$ & $24.30 \pm 6.017$ & 0.785 \\
\hline Nephrotic syndrome, n (\%) & $46(60.5)$ & $101(75.4)$ & 0.024 \\
\hline Serum creatinine $(\mu \mathrm{mol} / \mathrm{L})$ & $72.50(58.50-89.75)$ & $78.00(64.00-95.75)$ & 0.239 \\
\hline eGFR (ml/min /1.73) & 100.03 (81.31-111.37) & $92.67(74.71-106.63)$ & 0.041 \\
\hline Hypertension, n (\%) & $22(28.9)$ & $53(39.6)$ & 0.123 \\
\hline Diabetes, n (\%) & $14(10.4)$ & $9(11.8)$ & 0.756 \\
\hline Treatment & & & 0.135 \\
\hline ACEI/ARBs only, $n(\%)$ & $31(40.8)$ & $52(38.8)$ & \\
\hline Corticosteroids, n (\%) & $22(28.9)$ & $35(26.1)$ & \\
\hline Corticosteroids +CNI, n (\%) & $17(22.4)$ & $44(32.8)$ & \\
\hline Corticosteroids +CTX, n (\%) & \multicolumn{2}{|c|}{$3(2.2)$} & \\
\hline Spontaneous remission, $\mathrm{n}(\%)$ & $19(25.0)$ & $32(23.9)$ & 0.856 \\
\hline Treatment response, n (\%) & & & 0.852 \\
\hline Complete remission, n (\%) & $31(40.8)$ & $61(45.5)$ & \\
\hline Partial remission, n (\%) & $20(26.3)$ & $25(18.7)$ & \\
\hline No response, n (\%) & $25(32.9)$ & $48(35.8)$ & \\
\hline
\end{tabular}

IMN, idiopathic membranous nephropathy; eGFR, estimated glomerular filtration rate; ACEI/ARBs, angiotensin-converting enzyme inhibitors/angiotensin receptor blockers; CNIs, calcineurin inhibitors (including cyclophosphamide A and tacrolimus); CTX, cyclophosphamide

Table 4. Pathological characteristics of patients with IMN with and without IgM deposition 


\begin{tabular}{|c|c|c|c|}
\hline Parameters & $\begin{array}{l}\text { With IgM } \\
\text { deposition } \\
n=76\end{array}$ & $\begin{array}{l}\text { Without IgM } \\
\text { deposition } \\
n=134\end{array}$ & $P$ value \\
\hline Global sclerosis, n (\%) & $56(73.3)$ & $91(67.9)$ & 0.380 \\
\hline $\begin{array}{l}\text { Non-physiological global sclerosisê, } n \\
(\%)\end{array}$ & $16(21.1)$ & $25(18.7)$ & 0.674 \\
\hline FSGS, n (\%) & $21(27.6)$ & $18(13.4)$ & 0.011 \\
\hline Crescent, n (\%) & $4(5.3)$ & $11(8.2)$ & 0.426 \\
\hline Chronic interstitial fibrosis & & & 0.356 \\
\hline Absent, n (\%) & $28(36.8)$ & $37(27.6)$ & \\
\hline Mild, n (\%) & $41(53.9)$ & $85(63.4)$ & \\
\hline Moderate +Severer, $\mathrm{n}(\%)$ & $7(9.2)$ & $12(9.0)$ & \\
\hline Tubular atrophy & & & 0.969 \\
\hline $0-5 \%, n(\%)$ & $53(69.7)$ & $96(71.6)$ & \\
\hline $6 \%-25 \%, n(\%)$ & $19(25.0)$ & $31(23.1)$ & \\
\hline$\otimes 25 \%, \mathrm{n}(\%)$ & $4(5.3)$ & $7(3.3)$ & \\
\hline Vascular hyalinosis, n (\%) & $38(50.0)$ & $53(39.6)$ & 0.142 \\
\hline Membranous Churg's stageÖ & & & 0.478 \\
\hline Stage $\otimes /$ stage $\rrbracket-\rrbracket, n(\%)$, & $33(43.4)$ & $65(48.5)$ & \\
\hline$\geq$ Stage $\rrbracket, n(\%)$ & $43(56.6)$ & $69(51.5)$ & \\
\hline \multicolumn{4}{|l|}{ Immunofluorescence } \\
\hline IgG deposition, n (\%) & $76(100.0)$ & $133(99.3)$ & 1.000 \\
\hline IgG subclassification, n (\%) & $55(72.4)$ & $84(62.7)$ & 0.154 \\
\hline $\lg \mathrm{G} 1, \mathrm{n}(\%)$ & $51 / 55(92.7)$ & 79/84 (94.0) & 0.740 \\
\hline $\lg G 2, \mathrm{n}(\%)$ & $27 / 55(49.1)$ & 33/84 (39.3) & 0.254 \\
\hline $\lg G 3, n(\%)$ & $31 / 55(56.4)$ & $44 / 84(52.4)$ & 0.645 \\
\hline $\lg G 4, n(\%)$ & $52 / 55(94.5)$ & 83/84 (98.8) & 0.300 \\
\hline IgG4 dominant, n (\%) & $46 / 55(83.6)$ & 78/84 (92.9) & 0.087 \\
\hline IgA deposition, n (\%) & $28(36.8)$ & $34(25.4)$ & 0.080 \\
\hline C3 deposition, n (\%) & $65(85.5)$ & $116(86.6)$ & 0.834 \\
\hline
\end{tabular}




\begin{tabular}{|llll|}
\hline C3 deposition intensityU & & 0.656 \\
\hline Grade 1, n (\%) & $11(14.5)$ & $70(13.4)$ & \\
\hline Grade 2, n (\%) & $36(47.4)$ & $25(18.7)$ & \\
\hline Grade 3, n (\%) & $14(18.4)$ & $21(15.7)$ & \\
\hline Grade 4, n (\%) & $15(19.7)$ & $77(57.5)$ & 0.032 \\
\hline C1q deposition, n (\%) & $55(72.4)$ & $85(63.4)$ & 0.262 \\
\hline Glomerular PLA2R fluorescence, $\mathrm{n}(\%)$ & $54(71.1)$ & $79 / 85(92.9)$ & 0.484 \\
\hline Glomerular PLA2R positive, $\mathrm{n}(\%)$ & $52 / 54(96.3)$ & & \\
\hline
\end{tabular}

IMN, idiopathic membranous nephropathy; FSGS, focal segmental glomerular sclerosis; PLAR2, M-type phospholipase $\mathrm{A} 2$ receptor

ÊDefined as proportion of glomerulosclerosis exceeding ((age / 2) -10$) \times 100 \%$

ÖGlomerular IMN lesions were classified according to the Ehrenreich-Churg classification criteria ÜC3 subclass was determined using a semi-quantitative scale of 0 to 4: 0 , negative; 1 , weak staining; 2 , moderate staining; 3 , strong staining; 4 , glaring staining.

Table 5. Correlation of IgM deposition with FSGS lesions and C1q and C3 deposition

\begin{tabular}{|llll|}
\hline Parameters & $\mathrm{n}$ & $\mathrm{r}$ & $\mathrm{P}$ \\
\hline FSGS & $18.6 \%(39 / 210)$ & 0.175 & $\mathbf{0 . 0 1 1}$ \\
C1q & $66.2 \%(132 / 210)$ & 0.148 & $\mathbf{0 . 0 3 2}$ \\
C3 & $86.2 \%(181 / 210)$ & -0.014 & 0.835 \\
FSGS+ C1q & $11.0 \%(23 / 210)$ & 0.244 & 00.001 \\
FSGS+C3 & $16.7 \%(35 / 10)$ & 0.168 & $\mathbf{0 . 0 1 5}$ \\
FSGS+ C1q+C3 & $11.0 \%(23 / 210)$ & 0.244 & $\mathbf{0 0 . 0 0 1}$ \\
\hline
\end{tabular}

Note: All patients with FSGS + C1q deposition had C3 deposition.

FSGS, focal segmental glomerular sclerosis

Table 6. Relationship between IgM deposition and renal function prognosis 


\begin{tabular}{|llc|}
\hline & \multicolumn{1}{l}{ HR $(95 \% \mathrm{Cl})$} & P value \\
\hline & eGFR decline $\geq 5 \mathrm{ml} / \mathrm{min}$ & /1.73/year(cases=96) \\
\hline Model 1 & $2.063(1.369,3.109)$ & $\mathbf{0 . 0 0 1}$ \\
\hline Model 2 & $2.355(1.529,3.628)$ & $\square 0.001$ \\
\hline Model 3 & $2.324(1.508,3.582)$ & $\square 0.001$ \\
\hline Model 4 & $2.442(1.550,3.848)$ & $\square 0.001$ \\
\hline & & \\
\hline & eGFR decline $\geq 10 \%$ from baseline (cases=81) \\
\hline Model 1 & $1.939(1.244,3.022)$ & $\mathbf{0 . 0 0 3}$ \\
\hline Model 2 & $2.292(1.437,3.657)$ & $\mathbb{0} .001$ \\
\hline Model 3 & $2.278(1.427,3.636)$ & $\mathbf{0 . 0 0 1}$ \\
\hline Model 4 & $2.629(1.578,4.385)$ & 00.001 \\
\hline
\end{tabular}

Model 1: Unadjusted

Model 2: Adjusted for age and sex

Model 3: Adjusted for Model 1 covariates, proteinuria, and eGFR

Model 4: Adjusted for Model 2 covariates, glomerulosclerosis, FSGS lesions, crescents, chronic tubulointerstitial injury, vascular hyalinosis, membranous Churg's stage, C1q deposition, treatment, and treatment response

$\mathrm{HR}$, hazard ratio; $\mathrm{Cl}$, confidence interval; eGFR, estimated glomerular filtration rate; FSGS, focal segmental glomerular sclerosis

\section{Figures}




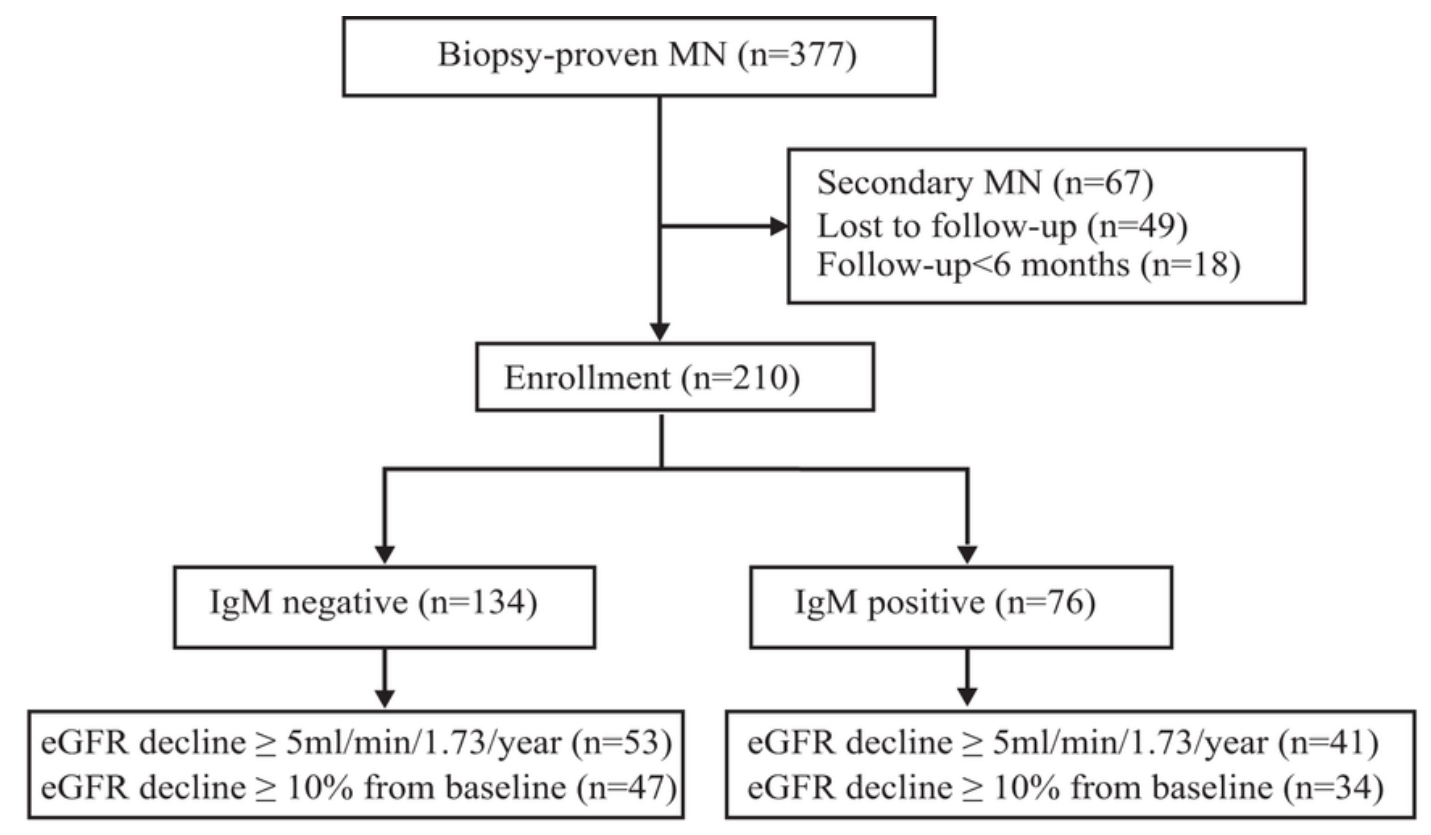

Fig. 1 Study flow, including patient enrollment and outcomes. MN, membranous nephropathy; eGFR, estimated glomerular filtration rate

\section{Figure 1}

See image above for figure legend
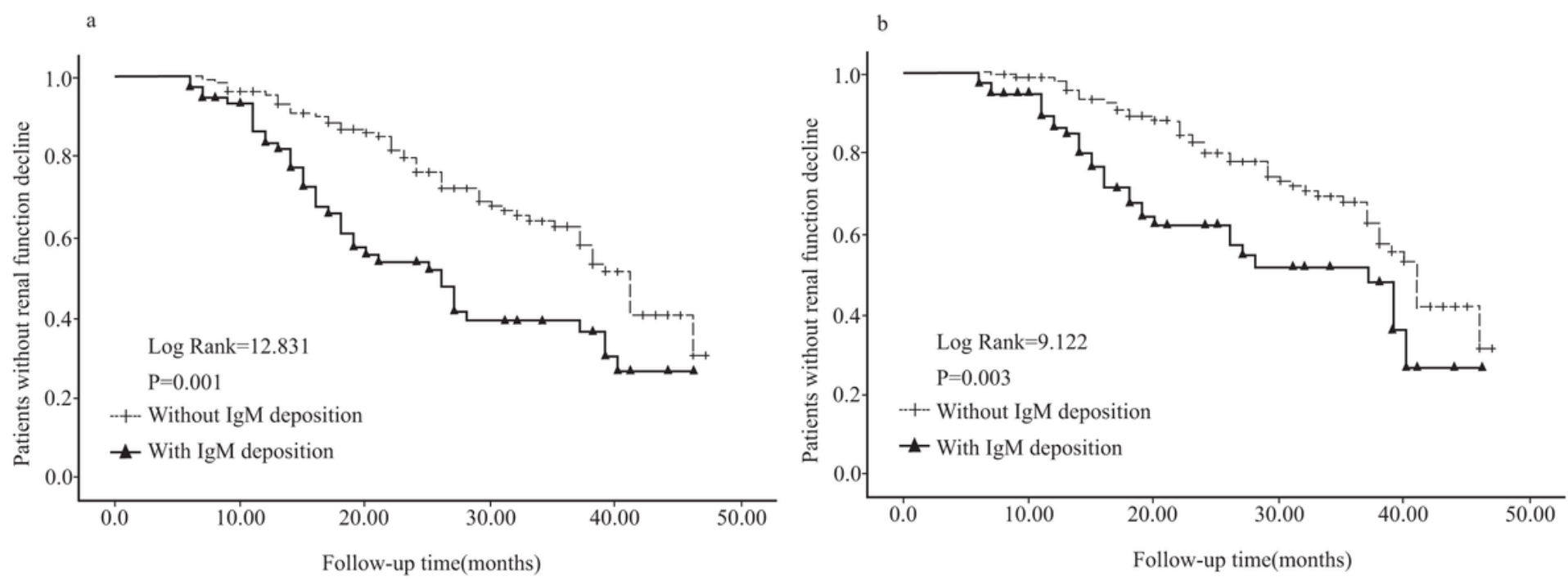

Fig. 2 Crude analyses of relationship between IgM deposition and renal function decline. Kaplan-Meier estimates of (a) eGFR decline of $\geq 5 \mathrm{~mL} / \mathrm{min} / 1.73 \mathrm{~m} 2$ per year and (b) eGFR decline of $\geq 10 \%$ from baseline. eGFR, estimated glomerular filtration rate

Figure 2 
See image above for figure legend

Page 21/21 